\title{
Photoelectrochemical Characterization and Durability Analysis of GaInPN Epilayers
}

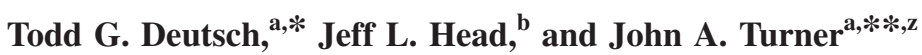 \\ ${ }^{a}$ National Renewable Energy Laboratory, Golden, Colorado 80401, USA \\ ${ }^{b}$ University of Arizona, Tucson, Arizona 85705, USA
}

Epilayer samples of GaInPN with a stoichiometry of $\mathrm{Ga}_{962} \mathrm{In}_{.038} \mathrm{P}_{976} \mathrm{~N}_{024}$ and $\mathrm{Ga}_{95} \mathrm{In}_{05} \mathrm{P}_{975} \mathrm{~N}_{025}$ were grown by metallorganic chemical vapor deposition and were photoelectrochemically characterized to determine their potential to photoelectrolyze water. The materials had direct transition bandgap energies of $\sim 2.0 \mathrm{eV}$, as determined by photocurrent spectroscopy; however, the measured flatband potentials were several hundred millivolts too negative for unbiased water splitting. Stability was assessed by operating the electrodes as photocathodes for $24 \mathrm{~h}$ and qualitatively evaluated by probing the amount of surface etching with a stylus profilometer. Quantitative evaluation was done by measuring the concentration of gallium in the corrosion test electrolyte using inductively coupled plasma mass spectrometry. Results were compared to similarly treated $\mathrm{GaP}_{\mathrm{P}} \mathrm{GaInP}_{2}$ samples for context.

(C) 2008 The Electrochemical Society. [DOI: 10.1149/1.2946478] All rights reserved.

Manuscript submitted January 15, 2008; revised manuscript received May 15, 2008. Published July 3, 2008.

A sustainable energy economy must be based on a renewable, nonpolluting energy source, and solar energy is the only resource that has the capacity to fully meet the need for carbon-free primary power for the future. ${ }^{1}$ Because solar energy is a variable resource and generates only electricity, it is necessary to store that energy and also provide an energy carrier for transportation; hydrogen is one material that can satisfy both requirements. Hydrogen is an ideal energy carrier because its consumption in a fuel cell generates only electricity, heat, and water and no pollutants. Direct solar-driven photoelectrolysis of water has great potential to provide hydrogen for a sustainable and environmentally benign hydrogen economy. Photoelectrochemical hydrogen generation would have advantages over a separated photovoltaic (PV) electrolyzer system because it would be able to achieve higher solar-to-hydrogen conversion efficiencies at lower cost. ${ }^{2}$

Direct photoelectrolysis systems require a semiconductor that simultaneously satisfies several material conditions relating to both the bulk and the interface with the electrolyte. First, the semiconductor must have a minimum bandgap sufficiently energetic to drive the electrolysis reaction. Under ideal conditions, water electrolysis requires $1.23 \mathrm{~V}$, but cathodic and anodic overpotentials require a material with a bandgap of at least $1.7 \mathrm{eV}^{3}$ A direct transition is preferred to reduce the amount of material necessary for complete optical absorption. The second criterion requires that the minority band-edge potential and the bulk Fermi level potential encompass the half reactions of the electrolysis reactions of interest. ${ }^{4}$ The semiconductor material must be stable against photocorrosion in an aqueous environment. Conversion of photons to chemical fuel must be highly efficient to keep material and system costs down. To date, no material satisfies all of these criteria. The most stable materials (oxides) have poor efficiencies, and the most efficient materials (III-V) lack stability. ${ }^{5,6}$ Several attempts have been made to both raise the efficiency of oxide materials and improve the stability of III-V materials, but they have met with only modest results. ${ }^{7-9}$

Previously, we showed that incorporating nitride into III-V semiconductors improved a number of the epilayer material's properties with respect to photoelectrolysis. ${ }^{10}$ Moderate amounts of nitrogen added to $\mathrm{GaP}\left(\mathrm{GaP}_{1-x} \mathrm{~N}_{x}, x<0.035\right)$ were found to improve absorption by inducing a direct transition at a lower energy than the native $\mathrm{GaP}$. Nitrogen also improved the stability of these photoelectrodes. However, the valence bandedges of GaPN and GaAsPN were determined to be too negative to be able to drive the anodic watersplitting half reaction without an applied bias. In addition, incorporating nitrogen into $\mathrm{GaP}$ causes a lattice contraction, leading to an

\footnotetext{
* Electrochemical Society Student Member.

** Electrochemical Society Active Member.

${ }^{\mathrm{z}}$ E-mail: John_Turner@nrel.gov
}

increasing mismatch between the epilayer and the substrate lattice constants as the nitride content increased. This lattice mismatch leads to defects in the material that can serve as recombination centers, thereby lowering efficiency. Defects at or near the surface can also lead to increased corrosion reactions that would not occur on a perfect crystalline surface. These issues effectively limit the amount of nitrogen that can be added in this manner and obscures any beneficial effects of increasing nitride content in the epilayer. There may be an ideal nitride content in this alloy that results in a perfect material for photoelectrolysis, but poor material quality stemming from the lattice mismatch masks the results.

The use of a lattice-expanding element in a quaternary nitride system can allow higher levels of nitrogen to be incorporated in the epilayer while maintaining a good lattice match. ${ }^{11}$ Arsenic has been used for lattice control; however as an amphoteric element, it reduces the stability of the alloy. ${ }^{10}$ Because indium is reported to be more stable in contact with the aqueous phase, for this work we used indium as a lattice expansion agent. ${ }^{12}$ Two compositions of $\mathrm{Ga}_{1-y} \mathrm{In}_{y} \mathrm{P}_{1-x} \mathrm{~N}_{x}$, specifically $\mathrm{Ga}_{.962} \mathrm{In}_{.038} \mathrm{P}_{.976} \mathrm{~N}_{.024}(2.4 \% \mathrm{~N})$ and $\mathrm{Ga}_{.95} \mathrm{In}_{.05} \mathrm{P}_{.975} \mathrm{~N}_{.025}(2.5 \% \mathrm{~N})$ were characterized to determine their potential to act as photoelectrodes in a solar-driven photolysis device. Similar material configurations have been considered for solidstate applications, such as heterojunction bipolar transistors or lightemitting diodes. ${ }^{13}$ The electronic and corrosion-resistant properties were compared to those of the well-characterized semiconductors $\mathrm{p}-\mathrm{GaP}$ and $\mathrm{p}-\mathrm{GaInP}_{2}$.

\section{Experimental}

The sample compositions $\mathrm{Ga}_{.962} \mathrm{In}_{.038} \mathrm{P}_{.976} \mathrm{~N}_{.024}$ and $\mathrm{Ga}_{95} \mathrm{In}_{.05} \mathrm{P}_{.975} \mathrm{~N}_{.025}$ were each grown by metallorganic chemical vapor deposition (MOCVD) on a $2 \times 2 \mathrm{~cm} \mathrm{Zn-doped} \mathrm{(001)} \mathrm{GaP} \mathrm{sub-}$ strate miscut $10^{\circ}$ toward the (110) face. ${ }^{14}$ The epilayers were $1 \mu \mathrm{m}$ thick and were deposited at a growth rate of $4 \mu \mathrm{m} / \mathrm{h}$. The pressure of the growth reactor was kept at $\sim 608$ Torr, and the substrate temperature was $650^{\circ} \mathrm{C}$ during sample growth. Hydrogen was used as a carrier gas, and the flow rates of the feedstock gases triethylgallium, trimethylindium, phosphine, and nitrogen trifluoride were used to control epilayer stoichiometry. The nitride epilayers were grown without the intentional addition of dopants. Compositions were estimated from X-ray diffraction (XRD) measurements of the lattice constant and Vegard's law (Table I). The p-GaP $(1 \mu \mathrm{m})$ was grown by MOCVD on a p-doped $\mathrm{GaP}$ substrate. The $\mathrm{p}$-GaInP 2 $(4.5 \mu \mathrm{m})$ was grown by MOCVD on a p-GaAs substrate. The GaP and $\mathrm{GaInP}_{2}$ epilayers were doped p-type with zinc $\left([\mathrm{p}]=1 \times 10^{17} \mathrm{~cm}^{-3}\right)$. Ohmic back contacts were made to $\mathrm{p}-\mathrm{GaP}$ substrates by electron-beaming palladium, followed by electroplat- 


\begin{tabular}{lccccc}
\hline $\begin{array}{l}\text { Table I. Elemental compositions of III-V semiconductors charac- } \\
\text { terized in this study. }\end{array}$ & \multicolumn{2}{c}{ Group III } & & \multicolumn{2}{c}{ Group V } \\
\cline { 2 - 3 } \cline { 5 - 6 } & $\mathrm{Ga}$ & $\mathrm{In}$ & & $\mathrm{P}$ & $\mathrm{N}$ \\
\hline Sample Name & 100 & & & 100 & \\
\hline GaP & 96.2 & 3.8 & & 97.6 & 2.4 \\
$\mathrm{GaInPN}(4 \%$ In) & 95 & 5 & & 97.5 & 2.5 \\
GaInPN (5\%In) & 51 & 49 & & 100 & \\
$\mathrm{GaInP}_{2}$ & & & & &
\end{tabular}

ing zinc, followed by another electron-beamed layer of palladium. The ohmic contact to p-GaAs was accomplished by electroplating gold.

Samples were received in wafer sizes of $\sim 4 \mathrm{~cm}^{2}$ and were cleaved with a diamond scribe to smaller chips of $\sim 0.2 \mathrm{~cm}^{2}$. The pieces were mounted to a coiled copper wire (ARCOR 16 gauge) with conducting silver paint (Ted Pella Conductive Silver 415) that was inserted into a 4 in. length of glass tubing. The edges and back of each electrode were covered with two layers of insulating epoxy. Loctite Hysol 9462 was the first layer used to cover the edges of the wafer chip. The second layer of epoxy, Loctite Hysol HP-120, covered the first layer of epoxy and all exposed elements. The two types of epoxy were required because Loctite 9462 is prone to chemical attack but has superior adhesion to the crystal surface. The electrodes had only $\sim 0.1 \mathrm{~cm}^{2}$ of the front surface of the semiconductor exposed to the environment.

XRD data were collected with a Bede D1 system, using $\theta-2 \theta$ scans with $0.5 \mathrm{~mm}$ slit width at the detector. The (004) reflection was analyzed as well as the (044) reflection to quantify strain.

Photocurrent spectroscopy, current-potential, open-circuit potential (OCP), Mott-Schottky, and corrosion testing and analysis were conducted using experimental systems and parameters described previously. ${ }^{10}$

The incident photon-to-current efficiency (IPCE) was determined using a monochromator system without light chopping nor lock-in detection. The slit width of the monochromator was $1 \mathrm{~mm}$, resulting in a bandwidth of $4 \mathrm{~nm}$. The dc current was measured with a Solartron 1287 potentiostat as the monochromator stepped through $5 \mathrm{~nm}$ intervals at $10 \mathrm{~s}$ periods. The $100 \mathrm{~W}$ tungsten lamp-monochromator output was measured similarly using a calibrated silicon photodiode (Hamamatsu). The IPCE at each wavelength was calculated by dividing the electrode photocurrent by the lamp output. No corrections for solution absorption were made.

\section{Results and Discussion}

Material quality.- Microscopic observation of the as-grown surfaces revealed few defects in these epilayers when compared to epilayers of GaPN with similar nitride content. ${ }^{10}$ The use of indium to counteract the lattice contraction from the incorporation of nitrogen appeared to yield a more perfect material. Minimal peak broadening was observed in the XRD spectra of the $\mathrm{GaInP}_{2}$ and GaInPN epilayers. XRD was not performed on $\mathrm{GaP}$ because the substrate and epilayer peaks are indistinguishable. Peak broadening is often correlated to the number of dislocations in a material, although it broadens for other reasons, such as epilayer thickness. A singlecrystal wafer typically has a full width half maximum (fwhm) $\sim 20$ arcsec, conversely, fwhm values over 100 arcsec might indicate problems with material quality. The fwhm values for $\mathrm{GaInP}_{2}$, GaInPN $(2.4 \% \mathrm{~N})$, and GaInPN $(2.5 \% \mathrm{~N})$ were 32,41 , and 31 arcsec, respectively. The analysis of the (044) reflections revealed that the nitride epilayers were $0 \%$ relaxed or completely strained to match the in-plane lattice constants, a condition typically observed for nearly lattice matched layers. Overall, XRD analysis revealed that all epilayers have excellent crystalline quality. ${ }^{15}$

Bandgap and transition type.- A direct transition is preferred for these materials because it typically leads to a high absorption

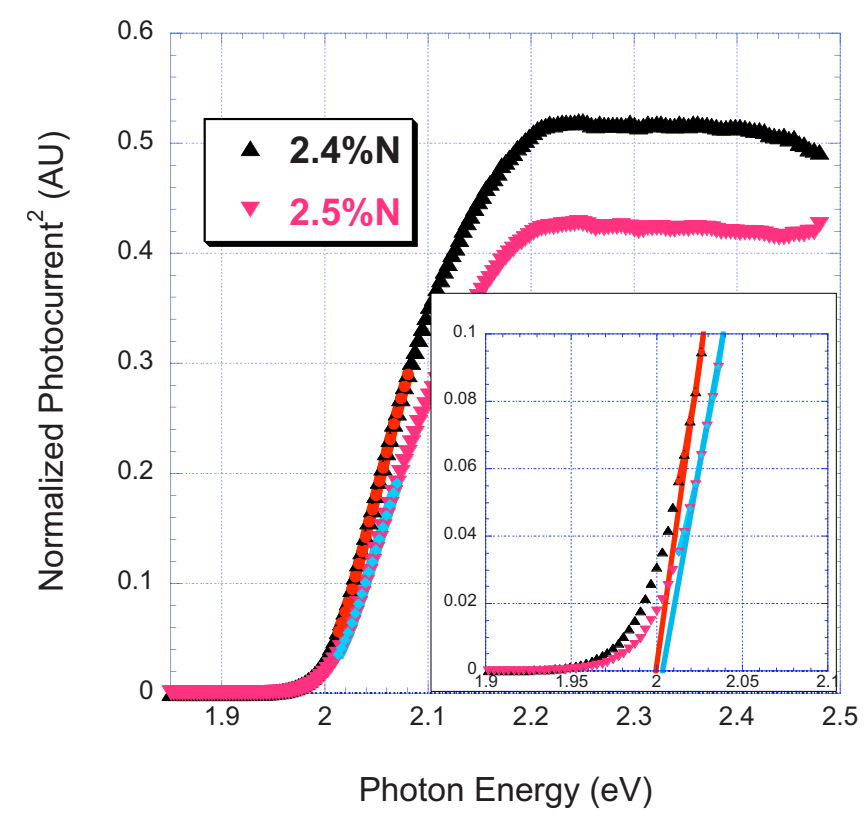

Figure 1. (Color online) Direct transition photocurrent spectra. Inset shows direct bandgap energy found by extrapolating linear portion of response to the $x$ axis.

coefficient, which has a number of benefits. A high absorption coefficient minimizes the amount of material necessary for complete absorption of the above-bandgap photons. Because photon penetration depth is greatly reduced, material purity requirements are not as stringent; photogenerated carriers have to traverse only a few microns before collection. The probability of encountering a defectrelated recombination center is diminished when the diffusion/drift distance is short.

Gallium phosphide is known to have an indirect bandgap at $2.26 \mathrm{eV},{ }^{16}$ whereas $\mathrm{GaInP}_{2}$ has a direct transition bandgap that can occur between 1.82 and $1.90 \mathrm{eV},{ }^{17}$ depending on growth conditions. Experimentally determined bandgaps for $\mathrm{GaP}(2.20 \mathrm{eV}$, indirect) and $\mathrm{GaInP}_{2}(1.81 \mathrm{eV}$, direct $)$ are close to the literature values. For the nitride samples, the square of photocurrent response was linear near the bandedge, indicating a direct electronic transition occurring at $2.00 \pm 0.01 \mathrm{eV}$ for both $2.4 \% \mathrm{~N}$ and $2.5 \% \mathrm{~N}$ (Fig. 1). This energy is great enough to provide the potential difference necessary to split water, including cathodic and anodic overpotentials $(\sim 1.7 \mathrm{~V})$ while still absorbing in the visible portion of the electromagnetic spectrum.

Incident photon-to-current efficiency.-Because any viable photoelectrolysis device must compete with PV/electrolysis for cost and efficiency, semiconductors used in photoelectrochemical (PEC) devices must have internal conversion efficiencies close to that of commercial PV devices, and the quantum yields for PV devices are often better than $90 \%$ (photon-to-electron) across the visible spectra. The equivalent measurement for PEC devices is IPCE, which is the same as the quantum yield but includes an electrochemical reaction at the semiconductor/electrolyte interface. To compete with PV/ electrolysis, IPCE should also be close to $90 \%$ across the visible portion of the spectrum. IPCE response can vary with applied potential and other environmental conditions (electrolyte $\mathrm{pH}$, redox couple, etc.); thus, across-the-board comparisons must be evaluated at identical operating point conditions to be valid.

The IPCE was measured for both GaInPN samples and compared to that for pure $\mathrm{p}-\mathrm{GaP}$ grown on a $\mathrm{GaP}$ substrate and $\mathrm{p}-\mathrm{GaInP}_{2}$ grown on a GaAs substrate (Fig. 2). The high conversion efficiency and sharp absorption edge, indicative of a direct transition, is apparent for the $\mathrm{p}-\mathrm{GaInP}_{2}$. The broader onset of absorption for $\mathrm{GaP}$ is indicative of an indirect transition. The absorption of the nitrides 


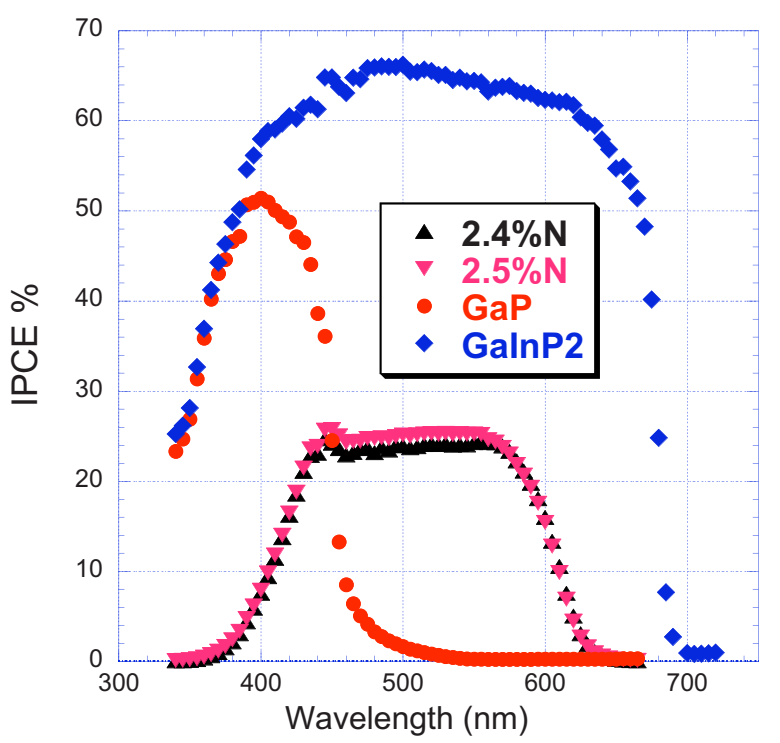

Figure 2. (Color online) IPCE for $\mathrm{GaP}, \mathrm{GaInP}_{2}$, and both compositions of GaInPN. IPCE data were taken at an applied bias of $-0.7 \mathrm{~V}$ vs the reference in $1 \mathrm{mM} \mathrm{Ru}\left(\mathrm{NH}_{3}\right)_{6}^{3+}$ in $\mathrm{pH} 2$ buffer. Illumination from the $100 \mathrm{~W}$ tungsten bulb was not chopped.

appears to fall in-between these two extremes, consistent with other reports $18,19,14$ of a "pseudo-direct" or "directlike" transition mode attributed to an impurity band ${ }^{20,21}$ or perturbed host states. ${ }^{22,23}$ Note in Fig. 2 the low IPCE values for the nitride materials and the drop-off of IPCE toward higher energies. The low IPCE at shorter wavelengths indicates a high surface recombination velocity. At higher photon energies, carriers are absorbed very close to the surface, where they are more susceptible to surface recombination. The relatively low plateau value for the nitride-containing samples is discouraging and indicates poor electronic properties, perhaps as a result of low mobilities or bulk recombination.

Current-potential analysis under simulated air mass (AM) 1.5 conditions is another way of assessing the conversion efficiencies possible for the three materials. As expected, the correlation between IPCE and the ability to generate photocurrent under broadband illumination is apparent (Fig. 3). Both the magnitude of saturated photocurrent and the shape of the response curve are further testimony to the state of the materials' electronic properties. Both $\mathrm{GaP}$ and $\mathrm{GaInP}_{2}$ exhibit ideal photocurrent response with a lightlimited photocurrent plateau that is relatively independent of applied bias. This type of response is indicative of materials with little bulk recombination. In contrast, the increase in photocurrent for the nitrides with additional reverse bias indicates high bulk recombination. The effect of increasing the bias is to stretch the depletion further into the bulk, where poor carrier transport properties (diffusion driven processes) can be overcome by field-aided collection (drift). The photocurrent limits for materials with a bandgap of $1.81 \mathrm{eV} \quad\left(\mathrm{GaInP}_{2}\right)$ and $2.00 \mathrm{eV} \quad(\mathrm{GaInPN})$ are 19.5 and $14.6 \mathrm{~mA} / \mathrm{cm}^{2}$, respectively. GaInPN falls well short of expectations due to surface and bulk recombinative losses; whereas $\mathrm{GaInP}_{2}$ exceeds the theoretical maximum because a $2.00 \mathrm{eV}$ reference cell was used to calibrate the light source, resulting in excess illumination intensity for a $1.81 \mathrm{eV}$ bandgap sample. Because $\mathrm{GaP}$ is too thin to be optically opaque (penetration depth exceeds sample thickness), it falls well short of the $9.6 \mathrm{~mA} / \mathrm{cm}^{2}$ theoretical maximum, despite the appearance of having more ideal transport properties.

Band alignment.- Band-edge potentials were determined by measuring the flatband potentials from two methods: the OCP under intense illumination and the potential of photocurrent onset. Both measurements were made in solutions of varying $\mathrm{pH}$, because reaction potentials and semiconductor band-edges positions are typically

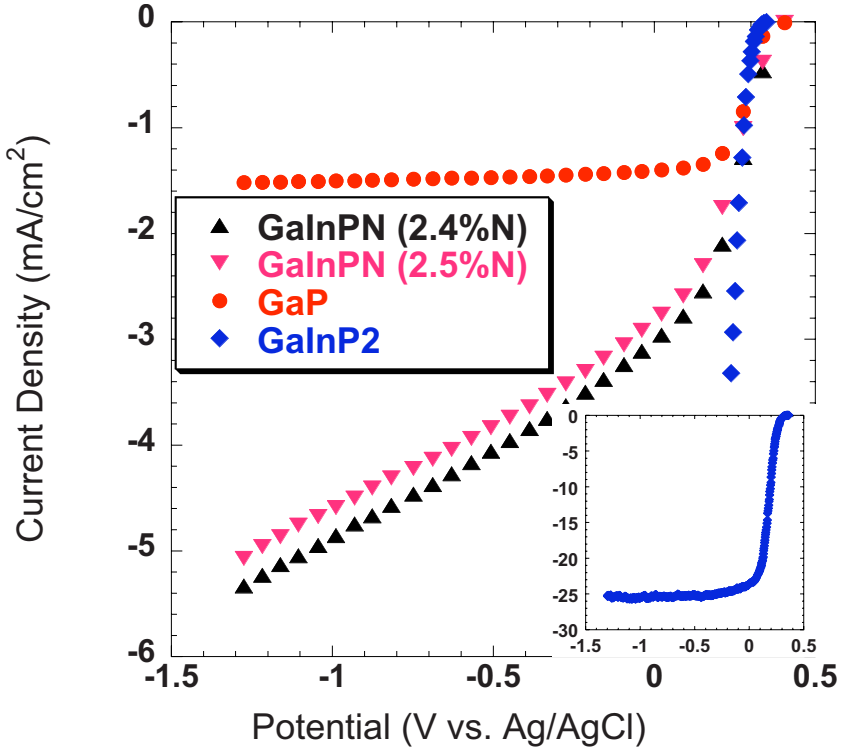

Figure 3. (Color online) Reverse bias current density vs potential curves $(25 \mathrm{mV} / \mathrm{s})$ in $3 \mathrm{M} \mathrm{H}_{2} \mathrm{SO}_{4}$ with surfactant, under $\mathrm{AM} 1.5$ broadband illumination from a $250 \mathrm{~W}$ tungsten lamp. Light intensity was set using a $2.0 \mathrm{eV}$ reference cell. Each electrode surface was treated with a platinum catalyst described elsewhere. ${ }^{10}$ The absence of a light-limited photocurrent plateau indicates recombination is a serious issue in the nitride epilayers.

$\mathrm{pH}$ dependent. OCP measurements were taken in the dark and under intense illumination. The shift in potential upon illumination was anodic, indicating p-type conductivity for all samples. The conduction band-edge potentials of both GaInPN sample compositions were sufficiently negative to drive hydrogen evolution; however, the potential of hole injection (Fermi level) was not anodic enough to generate oxygen from water at any $\mathrm{pH}$. The illuminated $\mathrm{OCP}$ values are plotted with the hydrogen and oxygen redox potentials with $\mathrm{pH}$ on the $x$ axis (Fig. 4). The GaP flatband potentials are included for reference. Photocurrent onset potentials are another method of estimating flatband potentials and were determined from electrodes that had a platinum surface treatment (Fig. 5). This was used to minimize

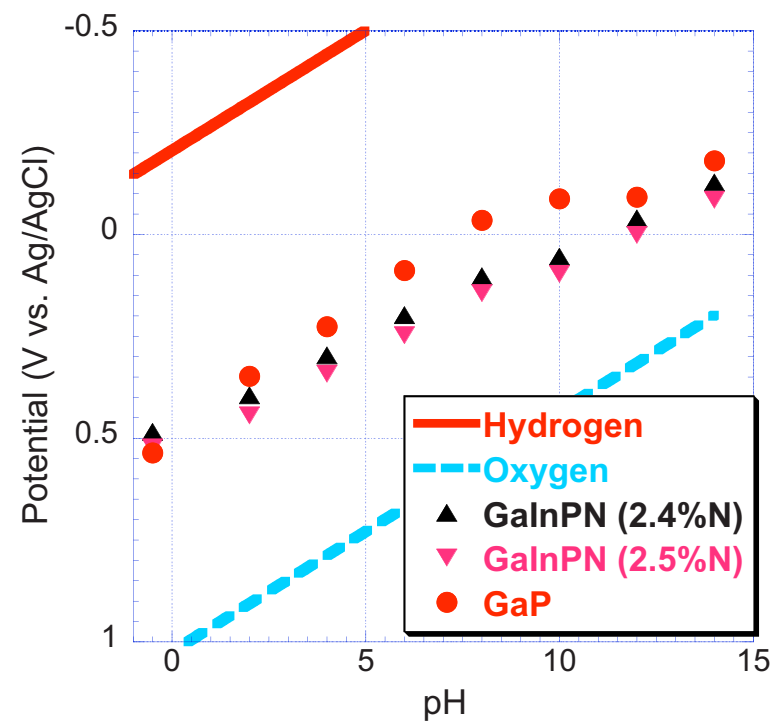

Figure 4. (Color online) Flatband potentials of GaP and GaInPN from illuminated OCP measurements. Data points must fall below the oxygen (dashed) line for these photocathodes to spontaneously split water upon illumination. 


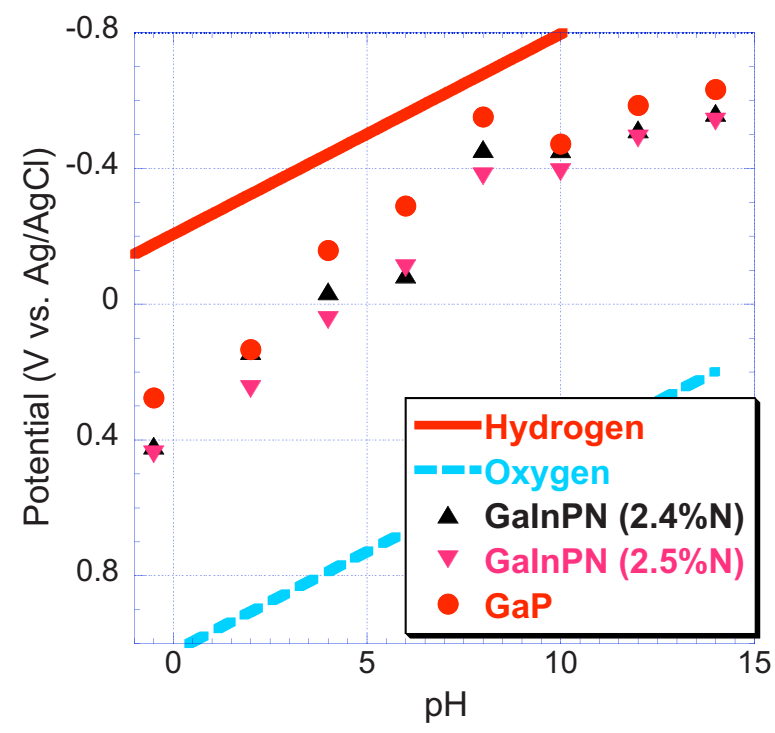

Figure 5. (Color online) Flatband potentials of GaP and GaInPN from photocurrent onset potential measurements. Data points must fall below the oxygen (dashed) line for these photocathodes to spontaneously split water upon illumination.

the error associated with the gas evolution overpotential. The cathodic photocurrent under reverse bias is another indication of p-type conductivity. The data plotted in Fig. 4 and 5 are average values of measurements taken on two electrodes from each sample composition. Typically, measured values of the two electrodes from each type of material varied by $<20 \mathrm{mV}$; thus, error bars are not included in the plots.

Both types of flatband measurements can have sources of error leading to flatband potential estimations that are slightly negative of the "true" values. A highly defective material can prevent the bands from flattening in OCP measurements as a result of recombination. This is a consideration here in light of the low ICPE values for the GaInPN samples. Flatband potentials based on photocurrent onset measurements can be complicated by reaction overpotential, even on a catalyzed electrode surface. The overpotential error in onset measurements is typically greater than that for the OCP (a few hundred millivolts) and could be invoked to justify the lack of congruence between measurements taken using each of the two methods. In light of the measurement errors, true flatband values are likely to be shifted toward the water oxidation potential, but not so much as to achieve overlap with the redox potentials.

Mott-Schottky analysis can also be used to determine flatband potentials, but the response for these III-V-N materials was not ideal and precluded its use here. The nonideality was manifested as noisy, nonlinear, hysteretic plots that had a dependence on frequency. Where a linear portion could be found, the fit led to aphysical flatband potentials (e.g., $5 \mathrm{~V}$ vs $\mathrm{Ag} / \mathrm{AgCl}$ ). Two-electrode short-circuit measurements using a platinum-black counter electrode confirmed that these materials are not capable of spontaneous photoelectrolysis because of the Fermi level mismatch. Most III-V materials are similarly deficient in both the magnitude and direction of the valence band mismatch ${ }^{24}$ and require an anodic bias on the counter electrode to photolyze water.

Stability. - The stability of these materials was evaluated by operating the electrodes galvanostatically for $24 \mathrm{~h}$ in $3 \mathrm{M}$ sulfuric acid that contained the surfactant Triton X-100. The surfactant was added to expedite bubble departure from the surface because large bubbles adhered to the surface lead to increased corrosion rates. ${ }^{10}$ Illumination at AM 1.5 (250 W tungsten) was used and the galvanostat current was $-5 \mathrm{~mA} / \mathrm{cm}^{2}$ (hydrogen evolution) over the duration of the test. After testing, the solutions were analyzed for Ga content using

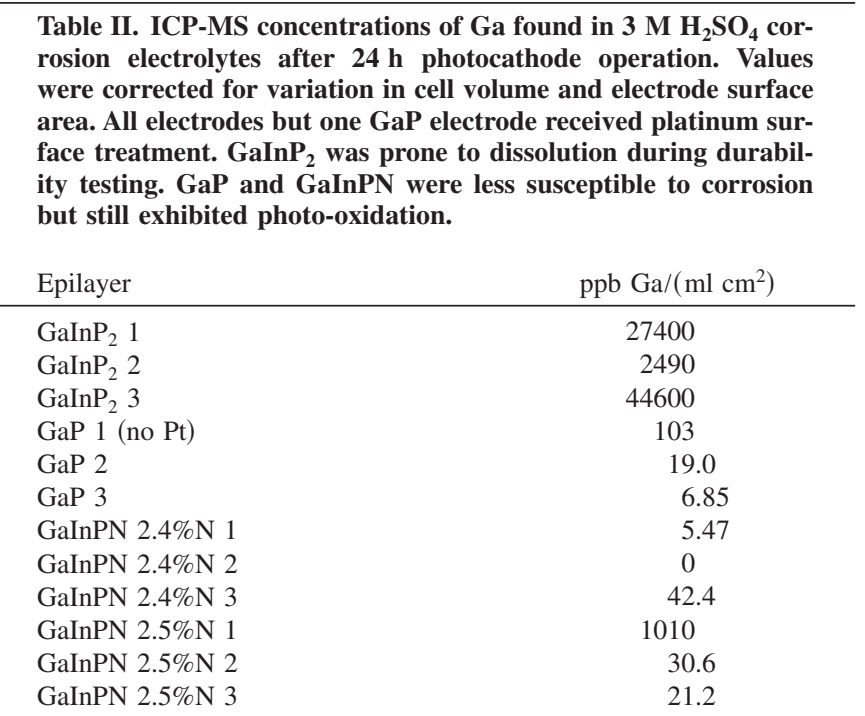

inductively coupled plasma mass spectrometry (ICP-MS). Results were compared to those for $\mathrm{GaP}$ and $\mathrm{GaInP}_{2}$ electrodes that received identical treatment (Table II). The wide spread of results for each sample set is most likely due to variation in the number of native defects present on the surface prior to the durability analysis. From the concentration of Ga determined from these samples, it is apparent that $\mathrm{GaP}$ and $\mathrm{GaInPN}$ are far more stable than $\mathrm{GaInP}_{2}$. Whether the nitride samples are more stable than pure $\mathrm{GaP}$ is not apparent from this data set. After durability testing, the photocurrent dropped to $\sim 10 \%$ of the predurability magnitude for all sample types.

Photomicroscopic analysis of the electrodes after durability testing, coupled with observations from stylus profilometry, also revealed some corrosion characteristics of the three material sets. The $\mathrm{GaInP}_{2}$ electrodes showed pitting of the surface on a nearly macroscopic scale, and pit diameters were $\sim 10 \mu \mathrm{m}$ (Fig. 6). The GaP electrodes exhibited fewer pits and were smaller, on the order of 1-3 $\mu \mathrm{m}$, and clustered around what appeared to be native defects. The corrosion mechanism of GaInPN was quite different from that of the nitride-free samples, where instead of being pitted, the surfaces appeared bumpy with protrusions having lateral dimensions up to $3 \mu \mathrm{m}$. No obvious pits were found in surveying the GaInPN surfaces. Profilometry was used to characterize the etching of the

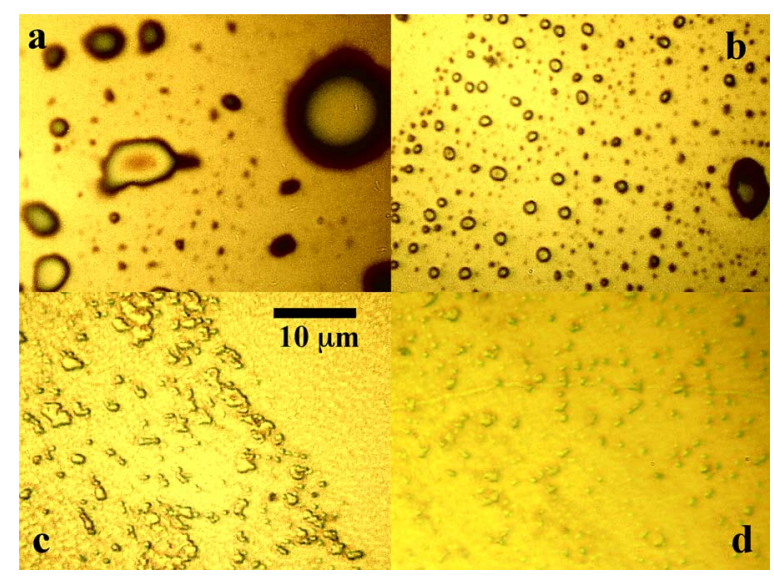

Figure 6. (Color online) Photomicroscopic images of durability electrode surfaces. (a) The $\mathrm{GaInP}_{2}$ electrode surface exhibits several large pits. (b) The $\mathrm{GaP}$ electrode had smaller pits clustered around larger defects. (c) Plateaus of the highly textured GaInPN $(2.4 \% \mathrm{~N})$ surface. (d) The GaInPN $(2.5 \% \mathrm{~N})$ had a similarly featured surface. 
samples after durability analysis and was carried out by dragging the stylus from the masked portion of the semiconductor sample into the region exposed to the electrolyte. Each $2 \mathrm{~mm}$ scan probed $\sim 1.5 \mathrm{~mm}$ of the exposed portion of the semiconductor. Three scans were taken from each side, and the profiles were highly variable. The average amount of material etched out during the $24 \mathrm{~h}$ test was $\sim 0.1 \mu \mathrm{m}$ for the $\mathrm{GaP}$ and $\mathrm{GaInPN}$ samples and $\sim 0.3 \mu \mathrm{m}$ for the $\mathrm{GaInP}_{2}$ sample.

\section{Conclusions}

The use of indium as a lattice-expanding agent allowed $>2 \%$ nitrogen incorporation in GaInPN epilayers with good lattice matching to a $\mathrm{GaP}$ substrate. The as-grown materials were p-type, and the bandgap for both compositions was found to be a direct transition with an energy of $\sim 2.0 \mathrm{eV}$. Incident photon-to-current efficiency measurements revealed low conversion efficiencies, indicating poor electronic properties or recombination processes limiting current generation in the devices. The flatband potentials of the III-V nitrides were too negative, resulting in a barrier for hole injection at the counter electrode, making it incapable of driving the oxygen reaction without an external bias. The stability of the nitrides was greater than that of $\mathrm{GaInP}_{2}$, as judged by ICP-MS analysis of the corrosion electrolytes, and similar to that of $\mathrm{GaP}$, but under these testing conditions, still prone to some corrosion. Although the corrosion resistance and conversion efficiencies of these samples are inadequate for a viable photoelectrolysis device, this quaternary nitride system does have some interesting characteristics, warranting further consideration. If the reasons for the low IPCE can be determined and addressed, it would useful to attempt to increase the stability by either surface treatments or solution additives.

\section{Acknowledgment}

The authors thank John Geisz and Sarah Kurtz for the semiconductor samples and Anna Duda for the ohmic contacts. We also acknowledge Fredrick Luiszer for the ICP-MS analysis. Thanks also to John Geisz for the XRD analysis and interpretation. This work was conducted at the National Renewable Energy Laboratory and funded by the U.S. Department of Energy Hydrogen Program under contract no. NXDJ-9-20638-23.

National Renewable Energy Laboratory assisted in meeting the publication costs of this article.

\section{References}

1. M. I. Hoffert, K. Caldeira, A. K. Jain, E. F. Haites, L. D. D. Harvey, S. D. Potter, M. E. Schlesinger, S. H. Schneider, R. G. Watts, T. M. L. Wigley, et al., Nature (London), 395, 881 (1998).

2. O. Khaselev, A. Bansal, and J. A. Turner, Int. J. Hydrogen Energy, 26, 127 (2001)

3. S. S. Kocha and J. A. Turner, Electrochim. Acta, 41, 1295 (1996).

4. A. J. Nozik, Annu. Rev. Phys. Chem., 29, 189 (1978).

5. A. J. Nozik, J. Cryst. Growth, 39, 200 (1977).

6. T. Bak, J. Nowotny, M. Rekas, and C. C. Sorrell, Int. J. Hydrogen Energy, 27, 991 (2002)

7. B. Marsen, E. L. Miller, D. Paluselli, and R. E. Rocheleau, Int. J. Hydrogen Energy, 32, 3110 (2007)

8. R. Noufi, A. J. Frank, and A. Nozik, J. Am. Chem. Soc., 103, 1849 (1981)

9. M. X. Tan, C. Newcomb, A. Kumar, S. R. Lunt, M. J. Sailor, B. J. Tufts, and N. S Lewis, J. Phys. Chem., 95, 10133 (1991).

10. T. G. Deutsch, C. A. Koval, and J. A. Turner, J. Phys. Chem. B, 110, 25297 (2006)

11. C. Skierbiszewski, P. Perlin, P. Wisniewski, W. Knap, T. Suski, W. Walukiewicz, W. Shan, K. M. Yu, J. W. Ager, E. E. Haller, et al., Appl. Phys. Lett., 76, 2409 (2000).

12. O. Khaselev and J. A. Turner, Corros. Sci., 42, 1831 (2000).

13. C. W. Tu, W. M. Chen, I. A. Buyanova, and J. S. Hwang, J. Cryst. Growth, 288, (2006).

14. J. F. Geisz, D. J. Friedman, W. E. McMahon, A. J. Ptak, A. E. Kibbler, J. M. Olson, S. Kurtz, C. Kramer, M. Young, A. Duda, et al., Paper NREL/CP-520-33545 presented at National Center for Photovoltaics and Solar Program Review Meeting, Denver, 2003

15. J. F. Geisz, Personal communication (2008)

16. M. R. Lorenz, G. D. Pettit, and R. C. Taylor, Phys. Rev., 171, 876 (1968)

17. J. M. Olson, S. R. Kurtz, A. E. Kibbler, and P. Faine, Appl. Phys. Lett., 56, 623 (1990).

18. Y. Zhang, B. Fluegel, M. Hanna, A. Duda, and A. Mascarenhas, Mater. Res. Soc Symp. Proc., 692, H2.1.1 (2002).

19. D. J. Friedmand and J. F. Geisz, Semicond. Sci. Technol., 17, 769 (2002).

20. Y. Zhang, B. Fluegel, and A. Mascarenhas, Phys. Rev. B, 62, 4493 (2000).

21. B. Fluegel, Y. Zhang, J. F. Geisz, and A. Mascarenhas, Phys. Rev. B, 72, 073203 (2005)

22. P. R. C. Kent and A. Zunger, Phys. Rev. B, 64, 115208 (2001).

23. Y. Zhang, A. Mascarenhas, and L.-W. Wang, Phys. Rev. B, 74, 041201 (2006)

24. A. J. Nozik and R. Memming, J. Phys. Chem., 100, 113061 (1996). 